\title{
Неорганічна хімія
}

\section{УДК 544:(344.3+015.35):546:(663’27’26):548.734 \\ ФАЗОВІ РІВНОВАГИ ТА КРИСТАЛІЧНА СТРУКТУРА СПОЛУК СИСТЕМИ ТЬ-В-С ПРИ $1270 \mathrm{~K}$}

\author{
В. Бабіжецький*, В. Левицький \\ Львівський національний університет імені Івана Франка, \\ вул. Кирила і Мефодія, 6, 79005 Львів, Украӥна \\ e-mail:v.babizhetskyy@googlemail.com
}

\begin{abstract}
Методами рентгенофазового і рентгеноструктурного аналізів енергодисперсійної та рентгенівської спектроскопії за довжиною хвилі (WDXS) побудовано ізотермічний переріз діаграми стану системи $\mathrm{Tb}-\mathrm{B}-\mathrm{C}$ при $1270 \mathrm{~K}$. Підтверджено існування восьми тернарних сполук. Визначено склади нових сполук $\sim \mathrm{Tb}_{2} \mathrm{BC}_{3}, \sim \mathrm{Tb}_{4} \mathrm{~B}_{5} \mathrm{C}_{18}$ та область гомогенності фази $\mathrm{Tb}_{5}(\mathrm{~B}, \mathrm{C})_{x}$, що належить до структурного типу $\mathrm{La}_{5} \mathrm{~B}_{2} \mathrm{C}_{6}$ й описано формулою $\mathrm{Tb}_{5}(\mathrm{~B}, \mathrm{C})_{x}, 8,3 \leq x \leq 9,2$ : $a=8,102(1), c=11,568(2) \AA$ (для $\mathrm{Tb}_{5} \mathrm{~B}_{2,1} \mathrm{C}_{6,2}$ ) та $a=8,054(1), c=12,335(4) \AA$ (для $\mathrm{Tb}_{5} \mathrm{~B}_{2,9} \mathrm{C}_{5,3}$ ). Кристалічну структуру сполуки $\mathrm{TbB}_{2} \mathrm{C}_{2}$ вперше уточнено рентгенівським методом монокристала: CT $\mathrm{DyB}_{2} \mathrm{C}_{2}$, ПГ $P 4 / \mathrm{mbm}, Z=2, a=5,337(1), c=3,575(1) \AA, R_{1}=0,019\left(w R_{2}=0,021\right)$ для 180 рефлексів з $I_{\mathrm{o}}>2 \sigma\left(I_{\mathrm{o}}\right)$.
\end{abstract}

Ключові слова: борокарбіди, потрійна система, фазові рівноваги, кристалічна структура.

DOI: https://doi.org/10.30970/vch.6001.012

\section{1. Вступ}

$\mathrm{Sc}, \mathrm{Y}, \mathrm{La}$ й лантаноїди, Ас й актиноїди проявляють найбільшу здатність серед металів утворювати тернарні борокарбіди. Утворені ними кристалічні структури характеризуються сітчастою будовою, у яких підрешітки атомів неметалів утворюють широкий топологічний ряд. Залежно від їхнього вмісту: від окремих атомів, що заповнюють порожнини, оточені атомами металів, до складних тривимірних каркасів [1]. Більшість тернарних борокарбідів рідкісноземельних металів (РЗМ, $R$ ) виявлено під час дослідження взаємодії компонентів у системах $R-\mathrm{B}-\mathrm{C}$, ізотермічні перерізи яких побудовано для $R=\mathrm{Sc}, \mathrm{Y}, \mathrm{La}, \mathrm{Ce}, \mathrm{Pr}, \mathrm{Eu}, \mathrm{Gd}, \mathrm{Ho}, \mathrm{Er}$ [2-10]. Проведені попередні дослідження системи Тb-B-C [11] підтвердили існування трьох відомих тернарних сполук $\mathrm{Tb}_{5} \mathrm{~B}_{2} \mathrm{C}_{6}$ (структурний тип (CT) $\mathrm{La}_{5} \mathrm{~B}_{2} \mathrm{C}_{6}$ ) [12], $\mathrm{Tb}_{5} \mathrm{~B}_{2} \mathrm{C}_{5}\left(\mathrm{CT} \mathrm{Sm}_{5} \mathrm{~B}_{2} \mathrm{C}_{5}\right)$ [13] та $\mathrm{TbB}_{2} \mathrm{C}_{2}\left(\mathrm{CT} \mathrm{DyB}_{2} \mathrm{C}_{2}\right)$ [14]. Під час подальших досліджень, крім відомих сполук, ми синтезували нові сполуки $\mathrm{Tb}_{10} \mathrm{~B}_{9} \mathrm{C}_{10}, \mathrm{~Tb}_{10} \mathrm{~B}_{7} \mathrm{C}_{10}$, $\mathrm{Tb}_{15} \mathrm{~B}_{4} \mathrm{C}_{14}, \mathrm{TbB}_{2} \mathrm{C}, \mathrm{Tb}_{2} \mathrm{~B}_{4} \mathrm{C}$ та $\mathrm{Tb}_{2} \mathrm{~B}_{2} \mathrm{C}_{3}$, що представляють нові структурні типи [15-20]. Для відомих тернарних борокарбідів Тербію кристалічні структури потребують уточнення. Попередній огляд кристалічних структур системи наведено у праці [1]. Поряд 3 цим, немає інформації стосовно діаграми фазових рівноваг у системі. Мета нашої праці - вивчити систему Tb-B-C у повному концентраційному інтервалі та побудувати ізотермічний переріз діаграми стану при $1270 \mathrm{~K}$.

(c) Бабіжецький В., Левицький В., 2019 
В. Бабіжецький, В. Левицький

ISSN 2078-5615. Вісник Львівського університету. Серія хімічна. 2019. Випуск 60. Ч. 1

\section{2. Матеріали та методика експерименту}

Зразки для дослідження синтезовано сплавлянням вихідних компонентів: Тербій (Alfa - Aesar, Johnson Matthey Company, чистотою $\geq 99,99$ мас. \%), графітовий порошок (Aldrich, $\geq 99,999$ мас. \%), кристалічний Бор (Н. C. Starck, Germany, чистотою $\geq 99,99$ мас. \%). Порошки графіту та Бору перед використанням дегазували прожарюванням за температури $1270 \mathrm{~K}$ та тиску $p<10^{-5}$ мбар. Стружку Р3М перемішували 3 порошками Бору та графіту, взятими у стехіометричних співвідношеннях, і пресували у сталевій прес-формі. Спресовані таблетки масою 1,000 г сплавляли в електродуговій або в індукційній печі в атмосфері очищеного аргону. Гомогенізувальний відпал сплавів, загорнутих у молібденову фольгу, проводили при $1270 \mathrm{~K}$ упродовж 800 год у вакуумованих кварцових ампулах. Відпалені зразки гартували в холодній воді, не розбиваючи ампул. Зразки для дослідження готували в атмосфері очищеного аргону, використовуючи посудини Шленка, шафу 3 інертною атмосферою та капіляри Ліндемана, заповнені і запаяні під аргоном.

\section{3. Результати досліджень та їх обговорення}

Ізотермічний переріз діаграми стану системи $\mathrm{Tb}-\mathrm{B}-\mathrm{C}$ побудовано на основі рентгенівського фазового аналізу (РФА) 83 зразків та аналізу мікроструктур деяких сплавів, результатів рентгенівської спектроскопії і який наведено на рис. 1. РФА проводили за дифрактограмами порошку (STOE STADI P, випромінювання МоК $\alpha_{1}$ ) 3 використанням комп'ютерної програми STOE WinXPOW [21]. Масив експериментальних даних отримано на автоматичному монокристальному дифрактометрі STOE IPDS I. Параметри кристалічної структури сполук методом порошку уточнювали за допомогою пакета програм WinCSD [22], а методом монокристала - за допомогою програми SHELX-97 [23] і пакета програм WinGX [24]. Для підтвердження фазового складу деяких концентраційних областей системи використовували метод енергодисперсійної рентгенівської спектроскопії за довжиною хвилі (WDXS) у поєднанні 3 растровим електронним мікроскопом TESCAN 5130 MM, обладнаного детекторами Oxford Si та Oxford INCA WAVE 700. Для визначення вмісту Бору та Тербію методом WDXS як стандарт використовували сполуку постійного хімічного складу $\mathrm{TbB}_{2} \mathrm{C}_{2}$.

За результатами дослідження відпалених при $1270 \mathrm{~K}$ зразків, синтезованих за вищеописаною методикою, у системі $\mathrm{Tb}-\mathrm{B}-\mathrm{C}$ ідентифіковано дванадцять тернарних сполук. Для десяти 3 них визначено кристалічну структуру. Уточнені значення параметрів елементарних комірок бінарних сполук подвійних систем $\mathrm{Tb}-\mathrm{B}$ та $\mathrm{B}-\mathrm{C}$, які обмежують потрійну, добре корелюють 3 літературними даними, наведеними у працях $[25,26]$. Після гомогенізаційного відпалу при $1270 \mathrm{~K}$ у зразках системи Тb-C підтверджено існування сполуки $\mathrm{Tb}_{3} \mathrm{C}_{4}$, виявленої при $1070 \mathrm{~K}$ під час дослідження трикомпонентної системи $\mathrm{Tb}-\mathrm{Cr}-\mathrm{C}$ [27], яка належить до СТ $\mathrm{Sc}_{3} \mathrm{C}_{4} . \mathrm{Tb}_{3} \mathrm{C}_{4}$ у литих зразках не виявлено. Для сполуки $\mathrm{Tb}_{2} \mathrm{C}_{3}\left(\mathrm{CT} \mathrm{Pu}_{2} \mathrm{C}_{3}\right)$ виявлено незначну область гомогенності, про що свідчить зміна параметрів елементарної комірки від $a=8,237(1)$ до $a=8,281(1) \AA$. Кристалографічні характеристики сполук, уточнених методом порошку, зібрано в табл. 1. За допомогою методу WDXS та РФА визначено склади двох нових сполук $\sim \mathrm{Tb}_{2} \mathrm{BC}_{3} \mathrm{i} \sim \mathrm{Tb}_{4} \mathrm{~B}_{5} \mathrm{C}_{18}$. Фаза складу $\sim \mathrm{Tb}_{2} \mathrm{BC}_{3}$ утворює рівноважні двофазні області зі сполуками $\mathrm{TbB}_{2} \mathrm{C}_{2}, \mathrm{TbC}_{2} \mathrm{Ta} \mathrm{Tb}_{5}(\mathrm{~B}, \mathrm{C})_{x}(8,3 \leq x \leq 9,2)$, а фаза 3 найбільшим вмістом Карбону $\sim \mathrm{Tb}_{4} \mathrm{~B}_{5} \mathrm{C}_{18}$ - зі сполуками $\mathrm{TbC}_{2}, \mathrm{TbB}_{2} \mathrm{C}_{2}, \mathrm{TbB}_{6}$ та Карбоном. 
В. Бабіжецький, В. Левицький

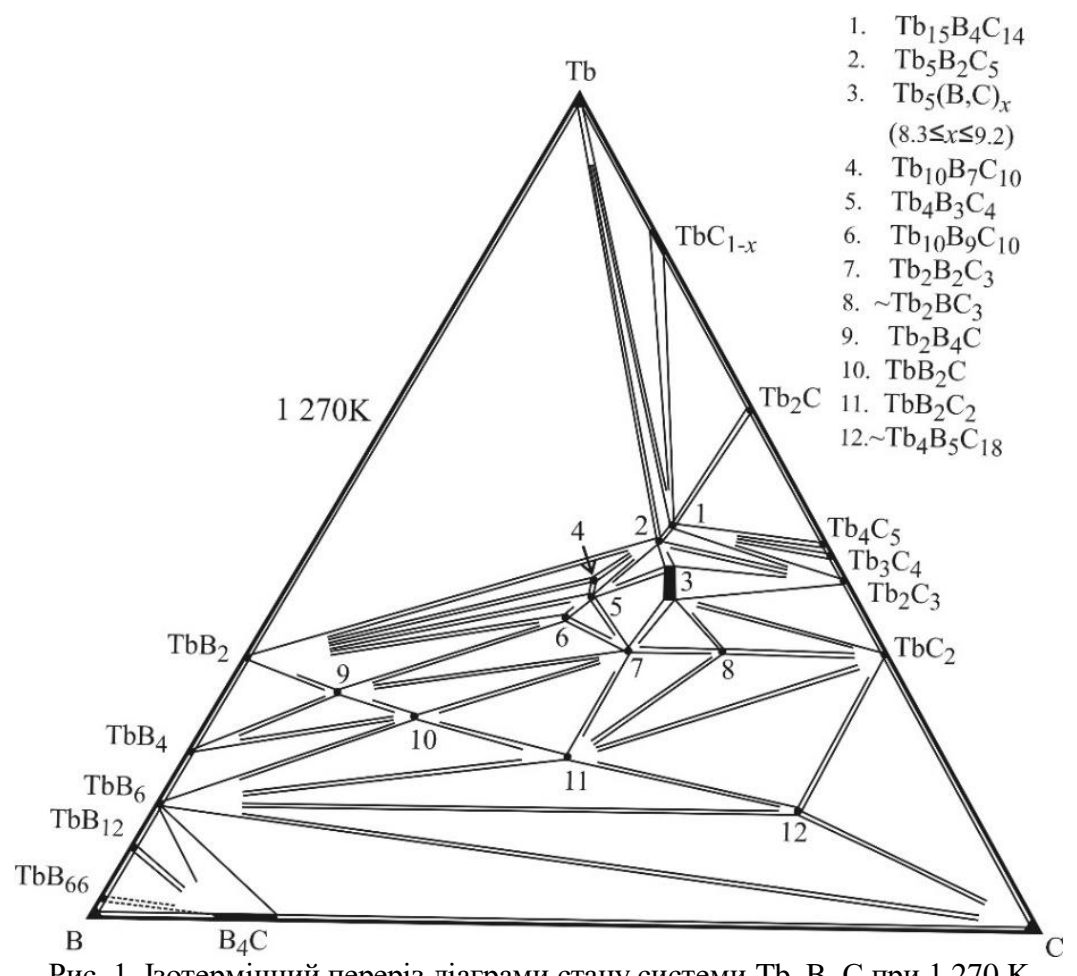

Рис. 1. Ізотермічний переріз діаграми стану системи Тb-B-C при $1270 \mathrm{~K}$

Fig. 1. Isothertmal section of the $\mathrm{Tb}-\mathrm{B}-\mathrm{C}$ phase diagram at $1270 \mathrm{~K}$

Кристалографічні характеристики сполук системи $\mathrm{Tb}-\mathrm{B}-\mathrm{C}$

Табличя 1

Table 1

Crystallographic data of ternary compounds in the $\mathrm{Tb}-\mathrm{B}-\mathrm{C}$ system

\begin{tabular}{|c|c|c|c|c|c|c|}
\hline \multirow{2}{*}{ Сполука } & \multirow{2}{*}{$\Pi \Gamma$} & \multirow{2}{*}{ CT } & \multicolumn{3}{|c|}{ Параметри елементарної комірки ( ()} & \multirow{2}{*}{ Літ. } \\
\hline & & & $a$ & $b$ & $c$ & \\
\hline 1. $\mathrm{Tb}_{15} \mathrm{~B}_{4} \mathrm{C}_{14}$ & $P 4 / m n c$ & $\mathrm{~Tb}_{15} \mathrm{~B}_{4} \mathrm{C}_{14}$ & $8,1491(8)$ & & $15,912(2)$ & $17, *$ \\
\hline 2. $\mathrm{Tb}_{5} \mathrm{~B}_{2} \mathrm{C}_{5}$ & $P 4 / n c c$ & $\mathrm{Sm}_{5} \mathrm{~B}_{2} \mathrm{C}_{5}$ & $8,1381(6)$ & & $10,861(1)$ & $13, *$ \\
\hline $\begin{array}{l}\text { 3. } \mathrm{Tb}_{5}(\mathrm{BC})_{x} \\
(8,3 \leq x \leq 9,2)\end{array}$ & $P 4 / n c c$ & $\mathrm{La}_{5} \mathrm{~B}_{2} \mathrm{C}_{6}$ & $\begin{array}{l}8,101(1)- \\
8,054(1)\end{array}$ & & $\begin{array}{l}11,568(2)- \\
12,335(4)\end{array}$ & $12, *$ \\
\hline 4. $\mathrm{Tb}_{10} \mathrm{~B}_{7} \mathrm{C}_{10}$ & $C 2 / c$ & $\mathrm{~Tb}_{10} \mathrm{~B}_{7} \mathrm{C}_{10}$ & $11,392(1)$ & $\begin{array}{l}11,240(2) \\
\beta=98,37(1)^{\circ}\end{array}$ & $23,704(3)$ & 16 \\
\hline 5. $\mathrm{Tb}_{4} \mathrm{~B}_{3} \mathrm{C}_{4}$ & $P \overline{1}$ & $\mathrm{Gd}_{4} \mathrm{~B}_{3} \mathrm{C}_{4}$ & $\begin{array}{l}3,6066(6) \\
\alpha=92,99(1)^{\circ}\end{array}$ & $\begin{array}{l}3,630(1) \\
\beta=96,77(1)^{\circ}\end{array}$ & $\begin{array}{l}11,8103(1) \\
\gamma=90,14(1)^{\circ}\end{array}$ & 16 \\
\hline 6. $\mathrm{Tb}_{10} \mathrm{~B}_{9} \mathrm{C}_{10}$ & $P 2{ }_{1} / c$ & $\mathrm{~Tb}_{10} \mathrm{~B}_{9} \mathrm{C}_{10}$ & $8,023(1)$ & $\begin{array}{l}23,954(1) \\
\beta=133,72(1)^{\circ}\end{array}$ & $11,317(2)$ & $15, *$ \\
\hline $\begin{array}{l}\text { 7. } \mathrm{Tb}_{2} \mathrm{~B}_{2} \mathrm{C}_{3} \\
\text { 8. } \sim \mathrm{Tb}_{2} \mathrm{BC}_{3}\end{array}$ & Cmmm & $\mathrm{Tb}_{2} \mathrm{~B}_{2} \mathrm{C}_{3}$ & $3,410(1)$ & $13,699(2)$ & $3,662(1)$ & $\begin{array}{l}20 * \\
*\end{array}$ \\
\hline 9. $\mathrm{Tb}_{2} \mathrm{~B}_{4} \mathrm{C}$ & Immm & $\mathrm{Dy}_{2} \mathrm{~B}_{4} \mathrm{C}$ & $3,2877(9)$ & $6,569(1)$ & $7,593(2)$ & $19, *$ \\
\hline 10. $\mathrm{TbB}_{2} \mathrm{C}$ & Pbam & $\mathrm{LuB}_{2} \mathrm{C}$ & $6,7844(3)$ & $6,7907(3)$ & $3,7883(2)$ & 28 , * \\
\hline $\begin{array}{l}\text { 11. } \mathrm{TbB}_{2} \mathrm{C}_{2} \\
\text { 12. } \sim \mathrm{Tb}_{4} \mathrm{~B}_{5} \mathrm{C}_{18}\end{array}$ & $P 4 / m b m$ & $\mathrm{DyB}_{2} \mathrm{C}_{2}$ & $5,3572(1)$ & & $3,5920(1)$ & $\begin{array}{c}14, * \\
*\end{array}$ \\
\hline
\end{tabular}

* Результати цієї праці, метод порошкової дифракції. 
В. Бабіжецький, В. Левицький

ISSN 2078-5615. Вісник Львівського університету. Серія хімічна. 2019. Випуск 60. Ч. 1

Область 3 високим вмістом Бору поблизу бінарного бориду $\mathrm{TbB}_{66}$ не досліджено. На рис. 2 зображено фотографії мікрошліфів, використаних для проведення кількісного елементного аналізу методом WDXS сполук $\mathrm{Tb}_{2} \mathrm{~B}_{2} \mathrm{C}_{3}$ та $\mathrm{Tb}_{2} \mathrm{~B}_{2} \mathrm{C}_{5}$. Фаза $\mathrm{TbB}_{2}$ практично не розчиняє третього компонента. Для фази зі структурним типом $\mathrm{La}_{5} \mathrm{~B}_{2} \mathrm{C}_{6}$ (просторова група (ПГ) P4/ncc) визначено область гомогенності, яку описано формулою $\mathrm{Tb}_{5}(\mathrm{~B}, \mathrm{C})_{x}(8,3 \leq x \leq 9,2)$. Результати цих досліджень наведено в табл. 2. Виявлено значну зміну параметрів елементарної комірки, яка зумовлена особливостями кристалічної структури CT $\mathrm{La}_{5} \mathrm{~B}_{2} \mathrm{C}_{6}$, які описані у працях $[4,28]$. Так, для складу $\mathrm{Tb}_{5} \mathrm{~B}_{2,1} \mathrm{C}_{6,2}$ об'єм елементарної комірки становить 759,35(1) $\AA^{3}$, а для $\mathrm{Tb}_{5} \mathrm{~B}_{2,9} \mathrm{C}_{5,3}-800,13(2) \AA^{3}$. Проведені дослідження кристалічної структури цієї сполуки рентгенівським методом монокристала свідчать про невпорядковане заповнення правильної системи точок $16 \mathrm{~g}$ атомами Бору та Карбону чотириатомних груп $\mathrm{BC}_{3}$ [12]. У системах La-B-C, Ce-B-C тa Pr-B-C [4-6] вміст Бору у сполуках цього СТ змінюється у дещо більших межах, ніж для $\mathrm{Tb}_{5}(\mathrm{BC})_{x}$, а в системі Er-B-C він є подібним, що свідчить також про вплив розмірного чинника на область гомогенності.
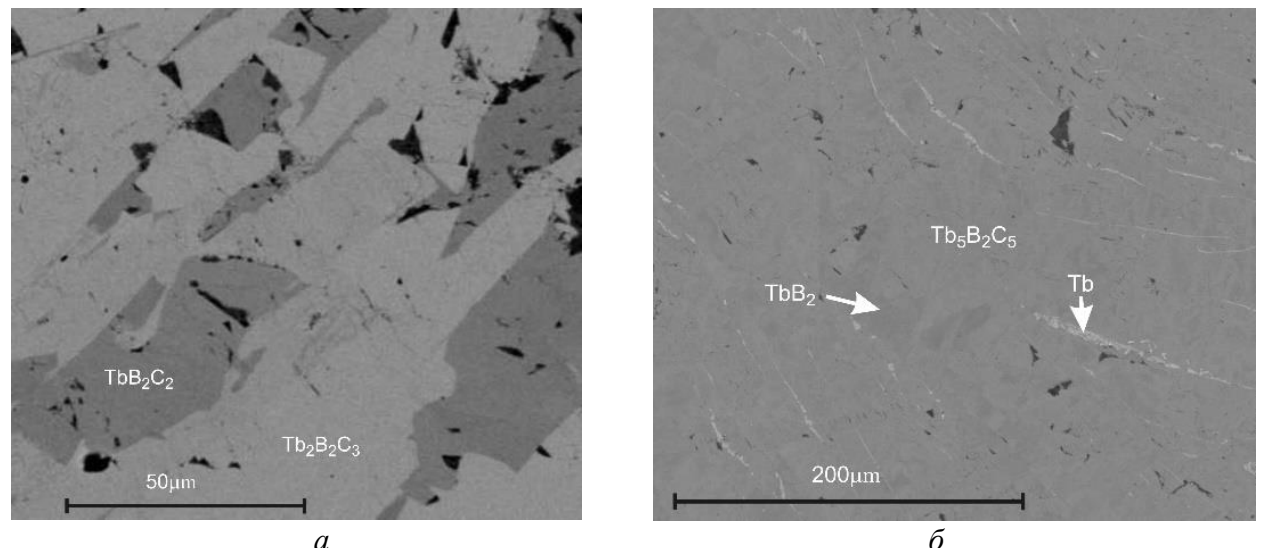

Рис. 2. Фотографії мікрошліфів зразків: $a) \mathrm{Tb}_{25} \mathrm{~B}_{30} \mathrm{C}_{45}$; б) $\mathrm{Tb}_{40} \mathrm{~B}_{35} \mathrm{C}_{25}$. Фазовий склад узгоджений за результатами РФА та WDXS

Fig. 2. Backscattered electron image of the annealed bulk samples: $a$ ) $\mathrm{Tb}_{25} \mathrm{~B}_{30} \mathrm{C}_{45}$; б) $\mathrm{Tb}_{40} \mathrm{~B}_{35} \mathrm{C}_{25}$. Phase composition in accordance with XRD and WDXS data

Таблиия 2

Зміна параметрів елементарної комірки твердого розчину* $\mathrm{Tb}_{5}(\mathrm{~B}, \mathrm{C})_{x}(8,3 \leq x \leq 9,2)$

Table 2

Crystallographic and chemical composition data for alloys $\operatorname{Tb}_{5}(\mathrm{~B}, \mathrm{C})_{x}(8.3 \leq x \leq 9.2)$

\begin{tabular}{|l|l|c|c|c|}
\hline Склад & $\begin{array}{l}\text { Параметри } \\
\text { елементарної комірки } \\
(a, c)\end{array}$ & $c / a$ & $V\left(\AA^{3}\right)$ & В, ат. \% \\
\hline $\mathrm{Tb}_{5} \mathrm{~B}_{2,1} \mathrm{C}_{6,2}{ }^{a}$ & $\begin{array}{l}8,101(1) \\
11,568(2)\end{array}$ & 1,427 & $759,35(1)$ & $16(1)$ \\
$\mathrm{Tb}_{5} \mathrm{~B}_{2,9} \mathrm{C}_{6,3}{ }^{\sigma}$ & $\begin{array}{l}8,054(1) \\
12,335(4)\end{array}$ & 1,531 & $800,13(2)$ & $22(1)$ \\
\hline
\end{tabular}

* Фазовий склад зразків: ${ }^{a} \mathrm{~Tb}_{5} \mathrm{~B}_{2,1} \mathrm{C}_{6,2}+\mathrm{Tb}_{5} \mathrm{~B}_{5} \mathrm{C}_{5} ;{ }^{6} \mathrm{~Tb}_{2} \mathrm{~B}_{2} \mathrm{C}_{3}+\mathrm{Tb}_{5} \mathrm{~B}_{2,9} \mathrm{C}_{5,3}+\sim \mathrm{Tb}_{2} \mathrm{BC}_{3}$. 
За результатами фазового аналізу сполука складу $\mathrm{TbB}_{2} \mathrm{C}_{2}$ утворює фазові рівноваги 3 чотирма тернарними фазами та двома бінарними (рис. 1). Оскільки раніше сполуку досліджували лише методом порошкової дифракції [14] та використано як стандарт під час дослідження сполук системи $\mathrm{Tb}-\mathrm{B}-\mathrm{C}$ методом

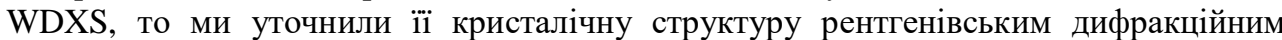
методом монокристала. Кристалохімічні характеристики сполуки та деталі зйомки наведено в табл. 3. Для дослідження використано модель кристалічної структури СТ $\mathrm{DyB}_{2} \mathrm{C}_{2}$ та уточнено ii в анізотропному наближенні параметрів зміщення атомів Тербію. Координати та відповідні параметри зміщення атомів у структурі сполуки $\mathrm{TbB}_{2} \mathrm{C}_{2}$ наведено в табл. 4.

Таблиия 3

Кристалохімічні характеристики, деталі знімання та уточнення структури сполуки $\mathrm{TbB}_{2} \mathrm{C}_{2}$

Crystal structure data and structure refinement of $\mathrm{TbB}_{2} \mathrm{C}_{2}$

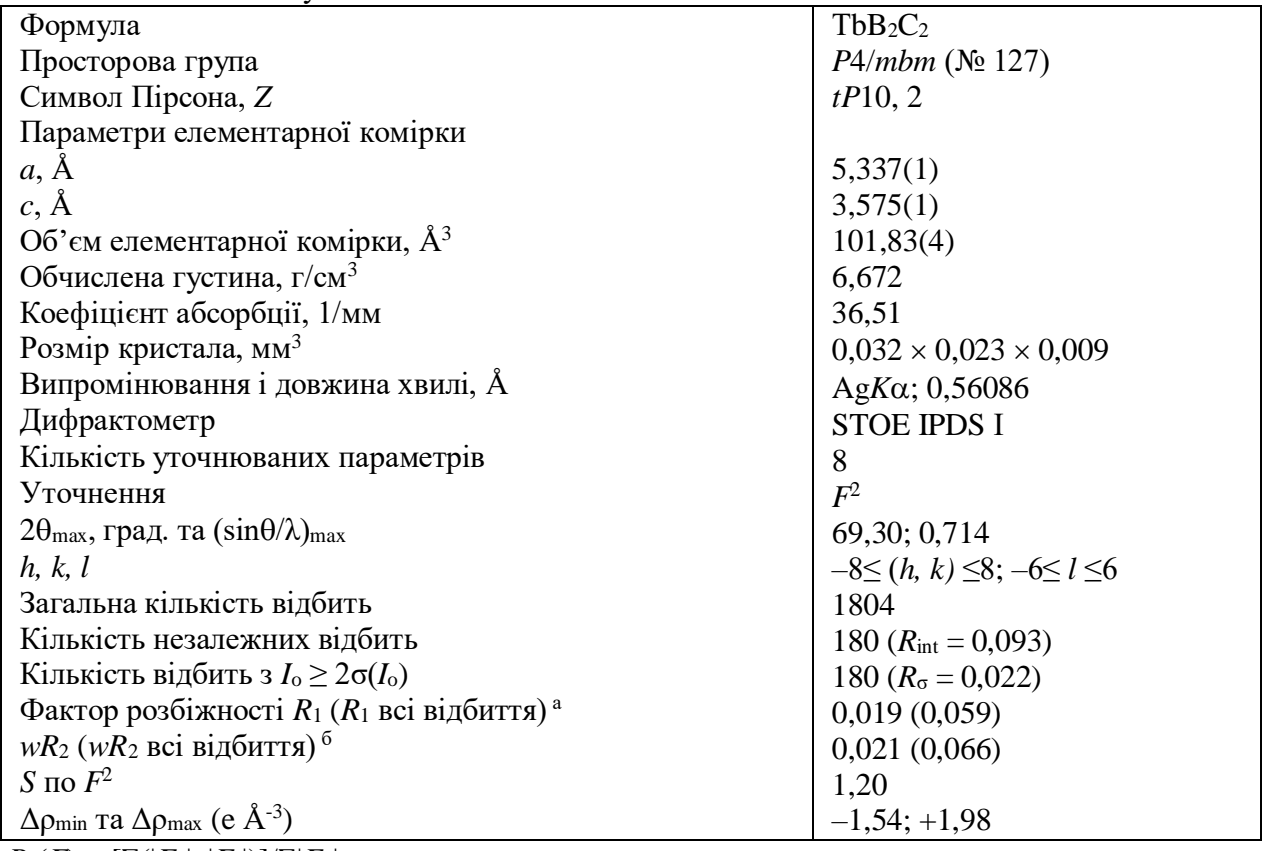

${ }^{\mathrm{a}} R_{1}(F)=\left[\Sigma\left(\left|F_{\mathrm{o}}\right|-\left|F_{\mathrm{c}}\right|\right)\right] / \Sigma\left|F_{\mathrm{o}}\right| ;$

${ }^{6} w R_{2}\left(F^{2}\right)=\left[\Sigma\left[w\left(F_{\mathrm{o}}{ }^{2}-F_{\mathrm{c}}{ }^{2}\right)^{2} / \Sigma\left[w\left(F_{\mathrm{o}}^{2}\right)^{2}\right]\right]^{1 / 2}\right.$, де $\left[w^{-1}=\sigma^{2}\left(F_{\mathrm{o}}\right)^{2}+(0,0108 P)^{2}+3,5600 P\right]$, де $P=\left(F_{\mathrm{o}}{ }^{2}+2 F_{\mathrm{c}}{ }^{2}\right) / 3$.

Координати та ізотропні параметри зміщення атомів у структурі $\mathrm{TbB}_{2} \mathrm{C}_{2}$

Таблиия 4

Positional and anisotropic displacement parameters for $\mathrm{TbB}_{2} \mathrm{C}_{2}$

Table 4

\begin{tabular}{|l|c|c|c|c|l|}
\hline \multicolumn{1}{|c|}{ Атом } & ПСТ & $x$ & $y$ & $z$ & \multicolumn{1}{c|}{$U_{\mathrm{eq}} / U_{\mathrm{iso}}, \AA^{2}$} \\
\hline $\mathrm{Tb}^{*}$ & $2 a$ & 0 & 0 & 0 & $0,0044(8)$ \\
$\mathrm{B}$ & $4 h$ & $0,361(1)$ & $0,139(1)$ & 0,5 & $0,005(2)$ \\
$\mathrm{C}$ & $4 h$ & $0,158(3)$ & $0,342(3)$ & 0,5 & $0,009(8)$ \\
\hline
\end{tabular}

$* U_{11}=0,044(8) ; U_{22}=0,044(8) ; U_{33}=0,024(9) ; U_{13}=U_{23}=U_{12}=0$. 


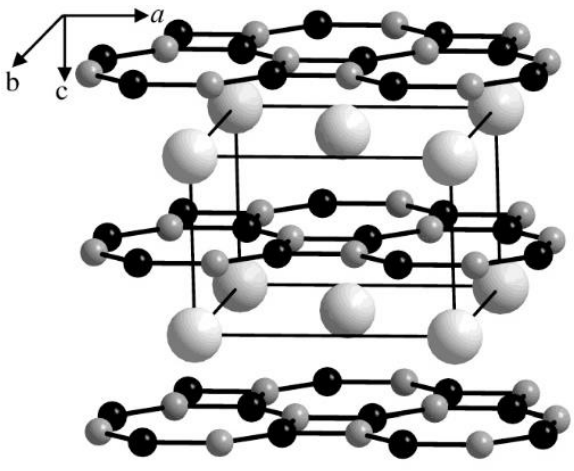

a)

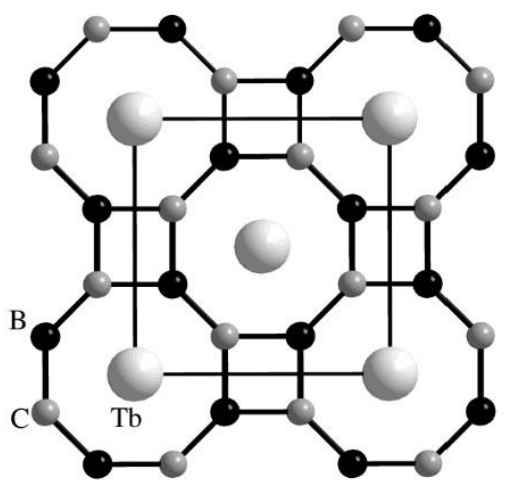

б)

Рис. 3. Розміщення бор-карбонових сіток уздовж осі $z$ у кристалічній структурі $\mathrm{TbB}_{2} \mathrm{C}_{2}(a)$ та 11 проекція на площину $x y$ (б). Виділено бор-карбонові кільця

Fig. 3. Side view (a) and projection on the (001) plane (б) of the $\mathrm{TbB}_{2} \mathrm{C}_{2}$ crystal structure. Boron-carbon rings are emphasized

За результатами монокристального дослідження підтверджено, що сполука має сталий стехіометричний склад і належить до $\mathrm{CT}_{\mathrm{DyB}} \mathrm{C}_{2}$ [29]. У структурі тернарного борокарбіду $\mathrm{TbB}_{2} \mathrm{C}_{2}$ плоскі сітки з чотири- $\left(\mathrm{B}_{2} \mathrm{C}_{2}\right)$ та восьмиатомних $\left(\mathrm{B}_{4} \mathrm{C}_{4}\right)$ кілець легких елементів розташовані безпосередньо одна над одною і не повернуті на $90^{\circ}$ у площині $x y$, як було запропоновано раніше у моделі кристалічної структури $\mathrm{LaB}_{2} \mathrm{C}_{2}$, поданої у праці [30]. Унаслідок цього в кристалічній структурі сполуки $\mathrm{TbB}_{2} \mathrm{C}_{2}$ не відбувається подвоєння параметра елементарної комірки $c$, як це виявлено для сполуки $\mathrm{CaB}_{2} \mathrm{C}_{2}$ [31]. Аналіз міжатомних віддалей легких елементів свідчить про нерегулярний зв'язок у площині двовимірної сітки $\left[\mathrm{B}_{2} \mathrm{C}_{2}\left(\mathrm{~B}_{4} \mathrm{C}_{4}\right)\right]_{\infty}^{2-}$. У бор-карбонових сітках кристалічної структури сполуки $\mathrm{TbB}_{2} \mathrm{C}_{2}$ наявні два типи В-C віддалей. Довші віддалі $\left(\delta_{\mathrm{B}-\mathrm{C}}=1,59(1) \AA\right)$ простежуються у чотири- та восьмиатомних кільцях 3 кутами між атомами у восьмиатомних кільцях $\angle$ В-C-B $=138,7(1)^{\circ}$ i $\angle_{\text {C-B-C }}=82,2(9)^{\circ}$. Короткі віддалі простежуються лише у восьмиатомних кільцях $\delta_{\mathrm{B}-\mathrm{C}}=1,53(1) \AA$ та кутами між легкими атомами $\angle_{\mathrm{B}-\mathrm{C}-\mathrm{B}}=82,2(9)^{\circ}$ та $\angle_{\mathrm{C}-\mathrm{B}-\mathrm{C}}=97,5(9)^{\circ}$. Атоми Тербію розміщені над восьмиатомними кільцями 3 дещо коротшими віддалями до атомів $\mathrm{C}\left(\delta_{\mathrm{Tb}-\mathrm{C}}=2,690(6) \AA\right)$, ніж до атомів В $\left(\delta_{\mathrm{Tb}-\mathrm{B}}=2,731(6) \AA\right)$, й утворюють псевдокубічну підгратку: віддалі $\mathrm{Tb}-\mathrm{Tb}$ у структурі $\mathrm{TbB}_{2} \mathrm{C}_{2}$ вздовж напряму [001] становлять 3,575(1) $\AA$ та 3,774(1) $\AA$ - вздовж [110].

\section{4. Висновки}

На основі рентгенофазового аналізу та аналізу мікроструктур, результатів рентгенівської спектроскопії побудовано ізотермічний переріз діаграми фазових рівноваг системи Tb-B-C при $1270 \mathrm{~K}$. За температури дослідження і використаній методиці отримання зразків у системі $\mathrm{Tb}-\mathrm{B}-\mathrm{C}$ ідентифіковано дванадцять тернарних сполук, для десяти 3 яких досліджено кристалічну структуру. Всі тернарні сполуки утворюються в області 15-45 ат. \% Тb. Уперше виявлено дві нові сполуки $\mathrm{Tb}_{2} \mathrm{BC}_{3}, \mathrm{~Tb}_{4} \mathrm{~B}_{5} \mathrm{C}_{18}$ та визначено їхні склади. Визначено область гомогенності для фази, що належить до структурного типу $\mathrm{La}_{5} \mathrm{~B}_{2} \mathrm{C}_{6}$, склад якої описано формулою $\mathrm{Tb}_{5}(\mathrm{BC})_{x}, 8,3 \leq x \leq 9,2$. В межах області гомогенності простежується зміна об'єму елементарної комірки кристалічної гратки від $759,35(1)$ до $800,13(2) \AA^{3}$. Кристалічну структуру сполуки $\mathrm{TbB}_{2} \mathrm{C}_{2}$ вперше вивчено методом монокристала та підтверджено іï ізоморфізм CT DyB $\mathrm{C}_{2}$. 


\section{5. Подяка}

Автори висловлюють щиру подяку професору А. Сімону та інженеру В. Дюппель (Інститут Дослідження Твердого Тіла ім. Макса Планка, м. Штутгарт, Німеччина) за проведення WDXS аналізу.

1. Babizhetskyy V., Bauer J., Gautier R., Hiebl K., Simon A., Halet J.-F. Chapter 302. Structural, electronic and physical properties of solid-state rare-earth boride carbides, in: Handbook on the Physics and Chemistry of the Rare Earths: including Actinides. Volume 53 (Eds. Bünzli J.-C. G., Pecharsky V. K.). North-Holland, Amsterdam, 2018. P. 145-269. DOI: https://doi.org/10.1016/bs.hpcre.2018.05.001

2. Shi Y., Leithe-Jasper A., Tanaka T. New ternary compounds $\mathrm{Sc}_{3} \mathrm{~B}_{0.75} \mathrm{C}_{3}, \mathrm{Sc}_{2} \mathrm{~B}_{1.1} \mathrm{C}_{3.2}$, $\mathrm{ScB}_{15} \mathrm{C}_{1.60}$ and subsolidus phase relations in the $\mathrm{Sc}-\mathrm{B}-\mathrm{C}$ system at $1700^{\circ} \mathrm{C} / / \mathrm{J}$. Solid State Chem. 1999. Vol. 148. P. 250-259. DOI: https://doi.org/10.1006/jssc.1999.8446

3. Bauer J., Nowotny H. Der Dreistoff Yttrium-Bor-Kohlenstoff // Monatsh. Chem. 1971. Vol. 102. P. 1129-1145. DOI: https://doi.org/10.1007/BF00909940

4. Babizhetskyy V., Simon A., Bauer J. Interaction of lanthanum with boron and carbon: phase diagram and structural chemistry // Monatsh. Chem. 2014. Vol. 145. P. 869-876. DOI: https://doi.org/10.1007/s00706-014-1172-2

5. Babizhetskyy V., Hembara M., Levytskyy V. Phase equilibria in Ce-B-C system at $1270 \mathrm{~K}$ and $970 \mathrm{~K} / /$ Proc. Shevchenko Sci. Soc. Chem. Sci. 2016. Vol. XLIV. P. 21-29 (in Ukrainian).

6. Babizhetskyy V., Simon A., Halet J.-F. Investigations in the ternary praseodymiumboron-carbon system: solid-state phase diagram and structural chemistry // Solid State Sci. 2015. Vol. 47. P. 73-77.

DOI: https://doi.org/10.1016/j.solidstatesciences.2014.12.008

7. Schwetz K. A., Hoerle M., Bauer J. Contribution to the system europium-boron-carbon // Ceramurgia Intl. 1979. Vol. 5. P. 105-109. DOI: https://doi.org/10.1016/0390-5519(79)90014-0

8. Ruiz D., Garland M. T., Saillard J.-Y., Halet J.-F., Bohn M., Bauer J. Electron probe microanalysis in the ternary Gd-B-C system // Solid State Sci. 2002. Vol. 4. P. 1173-1178. DOI: https://doi.org/10.1016/S1293-2558(02)01382-1

9. Bauer J., Venneguès P., Vergneau J. L. The ternary system holmium-boron-carbon; isothermal section at $1500{ }^{\circ} \mathrm{C} / /$ J. Less-Common Met. 1985. Vol. 110. P. 295-298. DOI: https://doi.org/10.1016/0022-5088(85)90335-2

10. Babizhetskyy V. Phase equilibria in Er-B-C system at $1270 \mathrm{~K} / /$ Visnyk Lviv Univ. Ser. Chem. 2017. Iss. 58. Pt. 1. P. 3-12 (in Ukrainian).

11. Babizhetskyy $V$., Mattausch Hj., Simon A. New ternary borocarbides in the $\mathrm{Tb}-\mathrm{B}-\mathrm{C}$ system // $\mathrm{X}^{\text {th }}$ Internat. Conf. Cryst. Chem. Intermet. Compds. Coll. Abstr. Lviv, Ukraine, 2007. P. 1.

12. Babizhetskyy $V$. Rare-earth boride carbides containing $\mathrm{BC}_{3}$ units: the crystal structure of $\mathrm{Tb}_{5} \mathrm{~B}_{2} \mathrm{C}_{6} / /$ Visnyk Lviv Univ. Ser. Chem 2013. Iss. 54. Pt. 1. P. 70-76 (in Ukrainian).

13. Bidaud E., Hiebl K., Hofmann R-D., Pöttgen R., Jardin C., Bauer J., Gautier R., Gougeon P., Saillard J.-Y., Halet J.-F. Structural, electronic and magnetic properties of ternary rare-earth metal borocarbides $R_{5} \mathrm{~B}_{2} \mathrm{C}_{5}(R=\mathrm{Y}, \mathrm{Ce}-\mathrm{Tm})$ containing $\mathrm{BC}_{2}$ "molecules" // J. Solid State Chem. 2000. Vol. 154. P. 286-295.

DOI: https://doi.org/10.1006/jssc.2000.8851 
14. Ohoyama K., Kaneko K., Indoh K., Yamauchi H., Tobo A., Onodera H., Yamaguchi Y. Systematic study on crystal structures in tetragonal $\mathrm{RB}_{2} \mathrm{C}_{2}$ ( $\mathrm{R}=$ Rare Earth) compounds // J. Phys. Soc. Japan. 2001. Vol. 70. P. 3291-3295.

DOI: https://doi.org/10.1143/JPSJ.70.3291

15. Babizhetskyy V., Hiebl K., Mattausch Hj., Simon A. New ternary boride carbides $R E_{10} \mathrm{~B}_{9+x} \mathrm{C}_{10-x}(R E=\mathrm{Gd}, \mathrm{Tb} ; x \approx 0.2)$ : infinite boron carbon branched chains // Z. Anorg. Allg. Chem. 2010. Vol. 636. P. 1229-1235. DOI: https://doi.org/10.1002/chin.201037014

16. Babizhetskyy V., Mattausch Hj., Simon A. Infinite and finite boron carbon branched chains: the crystal structures of new ternary boride carbides $\mathrm{RE}_{10} \mathrm{~B}_{7} \mathrm{C}_{10}$ and $\mathrm{RE}_{4} \mathrm{~B}_{3} \mathrm{C}_{4} / /$ Z. Anorg. Allg. Chem. 2009. Vol. 635. P. 737-742.

DOI: https://doi.org/10.1002/chin.200926017

17. Babizhetskyy V., Simon A., Mattausch Hj., Hiebl K., Zheng C. New ternary rare-earth metal boride carbides $R_{15} \mathrm{~B}_{4} \mathrm{C}_{14}(R=\mathrm{Y}, \mathrm{Gd}-\mathrm{Lu})$ containing $\mathrm{BC}_{2}$ units: crystal and electronic structures, magnetic properties // J. Solid State Chem. 2010. Vol. 183. P. 2343-2351. DOI: https://doi.org/10.1016/j.jssc.2010.07.046

18. Babizhetskyy V., Simon A., Hoch C., Hiebl K., Le Pollès L., Gautier R., Halet J.-F. Structural, electronic and magnetic properties of layered $R E \mathrm{~B}_{2} \mathrm{C}$ compounds $(R E=$ Dy, Tm, Lu $) / /$ J. Solid State Chem. 2012. Vol. 191. P. 121-128. DOI: https://doi.org/10.1016/j.jssc.2012.02.062

19. Babizhetskyy V., Zheng C., Mattausch Hj., Simon A. Ternary rare earth metal boride carbides containing two-dimensional boron-carbon network: The crystal and electronic structure of $R_{2} \mathrm{~B}_{4} \mathrm{C}(R-\mathrm{Tb}, \mathrm{Dy}, \mathrm{Ho}, \mathrm{Er}) / /$ J. Solid State Chem. 2007. Vol. 180. P. 3515-3520. DOI: https://doi.org/10.1016/j.jssc.2007.10.014

20. Babizhetskyy $V$., Mattausch Hj., Simon A. Crystal structure of terbium borocarbide $\mathrm{Tb}_{2} \mathrm{~B}_{2} \mathrm{C}_{3} / /$ Z. Naturforsch. 2008. Vol. 63b. P. 929-933.

DOI: https://doi.org/10.1515/znb-2008-0802

21. Stoe WinXPOW (Version 2.1). Stoe \& Cie GmbH. Darmstadt; Germany, 2004.

22. Akselrud L., Grin Y. WinCSD: software package for crystallographic calculations (Version 4) // J. Appl. Crystallogr. 2014. Vol. 47. P. 803-805. DOI: https://doi.org/10.1107/S1600576714001058

23. Sheldrick M. G. SHELXL-97: Program for the Refinement of Crystal Structures, University of Göttingen. Germany, 1997.

24. Farrugia L. J. WinGX suite for small molecule single-crystal crystallography // J. Appl. Crystallogr. 1999. Vol. 32. P. 837-838. DOI: https://doi.org/10.1107/S0021889899006020

25. Kuz'ma Yu. B., Chaban N. F. Binary and ternary systems containing boron. Moscow: Metallurgiya, 1990. 320 p. (in Russian).

26. Eremenko V. N., Velikanova T. Ya., Gordiichuk O. V. Carbides of rare earth metals: phase diagrams of the RE-C systems. Kiev: Naukova Dumka, 1993. 160 p. (in Russian).

27. Levytskyy V., Hembara M., Babizhetskyy V., Kotur B. System $\mathrm{Tb}-\mathrm{Cr}-\mathrm{C}$ at $800{ }^{\circ} \mathrm{C}$ : the phase equilibria and crystal structures of ternary compounds // Visnyk Lviv Univ. Ser. Chem. 2016. Iss. 57. Pt. 1. P. 23-33 (in Ukrainian).

28. Oeckler O., Mattausch Hj., Bauer J., Simon A. Über die Kristallstruktur der Phase $\mathrm{La}_{5} \mathrm{~B}_{2} \mathrm{C}_{6}$ // Z. Anorg. Allg. Chem. 2001. Vol. 627. P. 779-788. DOI: https://doi.org/10.1002/1521-3749(200104)627:4\%3C779::AID-ZAAC779\%3E3.0.CO;2-Y

29. Yamauchi H., Onodera H., Ohoyama K., Onimaru T., Kosaka M., Ohashi M., Yamaguchi $Y$. J. Antiferroquadropular ordering and magnetic properties of the tetragonal $\mathrm{DyB}_{2} \mathrm{C}_{2}$ compound // Phys. Soc. Japan. 1999. Vol. 68. P. 2057-2066.

DOI: https://doi.org/10.1143/JPSJ.68.2057 
30. Bauer J., Bars $O$. The crystal structure of the superconducting lanthanum boridecarbide $\mathrm{La}_{5} \mathrm{~B}_{2} \mathrm{C}_{6} / /$ J. Less-Common Met. 1983. Vol. 95. P. 267-274.

DOI: https://doi.org/10.1016/0022-5088(83)90520-9

31. Albert B., Schmitt $K$. $\mathrm{CaB}_{2} \mathrm{C}_{2}$ : Reinvestigation of a semiconducting boride carbide with a layered structure and an interesting boron/carbon ordering scheme // Inorg. Chem. 1999. Vol. 38. P. 6159-6163. DOI: https://doi.org/10.1021/ic9907821

\title{
PHASE EQUILIBRIA AND CRYSTAL STRUCTURE OF COMPOUNDS OF THE Tb-B-C SYSTEM AT $1270 \mathrm{~K}$
}

\author{
V. Babizhetskyy*, V. Levytskyy \\ Ivan Franko National University of Lviv, \\ Kyryla i Mefodia Str., 6, 79005 Lviv, Ukraine \\ e-mail:v.babizhetskyy@googlemail.com
}

The solid-state phase equilibria in the Tb-B-C system at $1270 \mathrm{~K}$ has been investigated by means of metallography, wavelength-dispersive X-ray spectroscopy (WDXS), powder and single crystal X-ray diffraction. Twelve ternary compounds were found to occur in the system. The existence of $\mathrm{TbB}_{2} \mathrm{C}_{2}, \mathrm{~Tb}_{5} \mathrm{~B}_{2} \mathrm{C}_{6}, \mathrm{TbB}_{2} \mathrm{C}, \mathrm{Tb}_{2} \mathrm{~B}_{4} \mathrm{C}, \mathrm{Tb}_{2} \mathrm{~B}_{2} \mathrm{C}_{3}, \mathrm{~Tb}_{10} \mathrm{~B}_{7} \mathrm{C}_{10}, \mathrm{~Tb}_{10} \mathrm{~B}_{9} \mathrm{C}_{10}, \mathrm{~Tb}_{4} \mathrm{~B}_{3} \mathrm{C}_{4}, \mathrm{~Tb}_{5} \mathrm{~B}_{2} \mathrm{C}_{5}$, and $\mathrm{Tb}_{15} \mathrm{~B}_{4} \mathrm{C}_{14}$ was confirmed. For the boundary compounds of the $\mathrm{Tb}-\mathrm{B}$ and $\mathrm{Tb}-\mathrm{C}$ systems no detectable extensions into the ternary region was found. Two new ternary compounds have been found, namely, $\sim \mathrm{Tb}_{2} \mathrm{BC}_{3}$ and $\sim \mathrm{Tb}_{4} \mathrm{~B}_{5} \mathrm{C}_{18}$. Powder $\mathrm{X}$-ray diffraction analysis of the samples close to $\sim \mathrm{Tb}_{4} \mathrm{~B}_{5} \mathrm{C}_{18}$ composition showed the large diffraction peaks may indicate a metal intercalation in the graphite matrix. The phase of $\mathrm{La}_{5} \mathrm{~B}_{2} \mathrm{C}_{6}$ structure type has a wide homogeneity range, described by formula: $\operatorname{Tb}_{5}(\mathrm{BC})_{x}(8.3 \leq x \leq 9.2)$. The structural arrangement of $\operatorname{Tb}_{5}(\mathrm{BC})_{x}(8.3 \leq x \leq 9.2)$ compound consists of a three-dimensional framework of rare-earth atoms resulting from the stacking of slightly corrugated two-dimensional squares, which lead to the formation of octahedral voids and distorted bicapped square antiprismatic voids. They are filled with isolated carbon atoms and twofold disordered $\mathrm{CBCC}$ units, respectively. The boron content in $[\mathrm{C}=\mathrm{B}-\mathrm{C}=\mathrm{C}]^{7-}$ varies from $16(1)$ to 22(1) at. \%, which leads to a decrease in charge as well as disorder. The overall charge of the anionic part seems to be compensated by introduction of single carbon atoms (C3) in $\mathrm{Tb}_{6}$ octahedra. The electron balance of $\mathrm{Tb}_{5} \mathrm{~B}_{2} \mathrm{C}_{6}$ can be written as $\left(\mathrm{Tb}^{3+}\right)_{5}\left(\mathrm{C}^{4-}\right)_{0.25}\left(\mathrm{CBCC}^{7-}\right)_{2}$. The crystal structure of $\mathrm{TbB}_{2} \mathrm{C}_{2}$ has been determined for the first time using single crystal X-ray diffraction data. It crystallizes in the tetragonal space group $P 4 / \mathrm{mbm}$, Pearson symbol $t P 10, a=5.337(1), c=3.575(1) \AA$, $R_{1}=0.019\left(w R_{2}=0.021\right)$ for 180 reflections with $I_{\mathrm{o}}>2 \sigma\left(I_{\mathrm{o}}\right)$.

$\mathrm{X}$-ray single crystal diffraction study showed that the four- and eight-member slightly puckered rings in the $\mathrm{TbB}_{2} \mathrm{C}_{2}$ structure consist of alternating $\mathrm{B}$ and $\mathrm{C}$ atoms. Two type of $\mathrm{B}-\mathrm{C}$ bonds are present: long bonds $\left(1.59(1) \AA\right.$, and $\mathrm{B}-\mathrm{C}-\mathrm{B}$ angle $\left.138.7(1)^{\circ}\right)$ common to the four- and eightmember rings and short bonds (1.53(1) $\AA$, and $\mathrm{B}-\mathrm{C}-\mathrm{B}$ angle $\left.82.2(9)^{\circ}\right)$ unique to the eight-member rings. $\mathrm{Tb}$ atoms are located above the centers of the eight-member rings and have slightly larger contacts to $\mathrm{C}\left(\delta_{\mathrm{Tb}-\mathrm{C}}=2.690(6) \AA\right)$, than to $\mathrm{B}\left(\delta_{\mathrm{Tb}-\mathrm{B}}=2.731(6) \AA\right)$ atoms, and form a pseudo-cubic sublattice: the $\mathrm{Tb}-\mathrm{Tb}$ distances in the structure of $\mathrm{TbB}_{2} \mathrm{C}_{2}$ are 3.575(1) $\AA$ along [001] direction, and 3.774(1) $\AA$ - along [110]. These are comparable with other Ln-Ln distances obtained from neutron diffraction study possessing lanthanoid contraction.

Keywords: borocarbides, ternary system, phase equilibria, crystal structure.

Стаття надійшла до редколегії 31.10 .2018

Прийнята до друку

23.01.2019 